\title{
Beamforming Using Passive Nested Arrays of Sensors
}

\author{
Piya Pal and P. P. Vaidyanathan \\ Dept. of Electrical Engineering, MC 136-93 \\ California Institute of Technology, Pasadena, CA 91125, USA \\ E-mail: piyapal@caltech.edu,ppvnath@systems.caltech.edu
}

\begin{abstract}
A novel approach to beamforming using a new class of sensor arrays is proposed, which can increase the achievable degrees of freedom significantly beyond the conventional limits obtained from uniform linear arrays (ULA). This class of arrays is named as "nested arrays" since they are obtained by nesting two or more ULAs with increasing inter-sensor spacing. Using the second order statistics of the signal received by such an array in a novel way, it is possible to perform beamforming with $O\left(N^{2}\right)$ degrees of freedom using only $O(N)$ physical elements. This kind of beamforming will be shown to be essentially non linear in nature and theoretically, it is capable of nulling the effect of noise provided enough snapshots are available.

Index Terms - Beamforming, Co-array, Degrees of Freedom, Jammer, Non Uniform Arrays.
\end{abstract}

\section{INTRODUCTION}

Beamforming using antenna arrays is the spatial analogue of temporal filtering which forms the core of all signal processing applications [9], [4]. It is widely used to improve detection and estimation performance by enhancing the signal to interference and noise ratio (SINR) in diverse applications like radar, sonar, acoustics, smart antennas for communication etc. Traditionally, beamforming is performed using an array of uniformly spaced antennas because such an array performs uniform sampling of the space, making it easier to directly apply the various filtering techniques of traditional digital signal processing. This kind of beamforming is linear with respect to the amplitude of the impinging signals. However the degrees of freedom obtainable from a ULA of $N$ sensors, is $N$.

In this paper, we propose a novel "non linear" approach to beamforming which is capable of increasing the degrees of freedom much beyond the conventional limit of $N$. This approach is based on two main concepts: (1) Use of a novel "nested array" structure (instead of a ULA), and (2) Performing spatial filtering with respect to the signal powers instead of the amplitudes. With this nested array, it is possible to realize a longer "virtual array" which is identical to the difference co-array [10] of the original array, by using the second order statistics of the received signals. The co-array of the nested array structure, as we shall demonstrate, has $O\left(N^{2}\right)$ degrees of freedom though the original array has only $O(N)$ sensors and hence we can effectively perform beamforming with a considerable increase in degrees of freedom. This increase in degrees of freedom can be used to obtain better spatial resolution, suppression of more jammers and so forth.

A somewhat similar approach to beamforming was explored in [2] where specific examples of some special arrays were considered instead of suggesting a general class to systematically increase the degrees of freedom. Our proposed nested array, however, constitutes a broader and more general class of arrays which can be constructed in an easy and systematic fashion. We shall also provide the exact degrees of freedom

${ }^{1}$ Work supported in parts by the ONR grant N00014-08-1-0709, and the California Institute of Technology. associated with each nesting strategy and optimize across all possible strategies to maximize the degrees of freedom [7]. In [1], a longer "virtual array" was realized through active sensing with a MIMO radar but in our case, the increase in degrees of freedom will be obtained in a passive setting. As we shall show, this beamforming spatially filters the powers of the sources (instead of their amplitude) and hence it is inherently non-linear in nature. Another major advantage of the proposed approach to beamforming is that, assuming perfect estimation of the signal covariance matrix, it can eliminate noise, which is never possible using conventional linear approach to beamforming. An application of this nested array in passive DOA estimation with increased degrees of freedom is discussed in another submission [6].

The rest of the paper is organized as follows. In Section II, we deduce the non-linear signal model and define the new non linear approach to beamforming. In Section III we propose a new class of nested arrays and demonstrate how they are capable of providing significant increase in degrees of freedom. In Section IV, we develop the new beamforming technique for realizing both deterministic beampatterns and nulling of jammers and noise. The performance of the proposed beamformer is validated through numerical examples in Section V.

\section{A New Approach to BeAmforming}

Consider a $N$ element (possibly non uniform) antenna array. Let $\mathbf{a}(\theta)$ be the $N \times 1$ steering vector corresponding to the direction $\theta$. Let us assume $D$ narrowband sources impinging on this array from directions $\left\{\theta_{d}, d=1,2, \cdots, D\right\}$, with powers $\left\{\sigma_{d}^{2}, d=1,2, \cdots, D\right\}$ respectively. The received signal can be written as

$$
\mathbf{x}[k]=\mathbf{A} \mathbf{s}[k]+\mathbf{n}[k]
$$

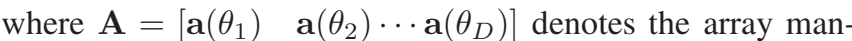
ifold matrix and $\mathbf{s}[k]=\left[s_{1}[k] \quad s_{2}[k] \cdots s_{D}[k]\right]^{T}$ denotes the source signal vector. The noise $\mathbf{n}[k]$ is assumed to be temporally and spatially white, and uncorrelated from the sources. We also assume the sources to be temporally uncorrelated so that the source autocorrelation matrix of $\mathbf{s}[k]$ is diagonal. The autocorrelation matrix of $\mathbf{x}[k]$ can be written as:

$$
\begin{aligned}
\mathbf{R}_{\mathbf{x x}} & =E\left[\mathbf{x x}^{H}\right] \\
& =\mathbf{A} \mathbf{R}_{\mathbf{S S}} \mathbf{A}^{H}+\sigma_{n}^{2} \mathbf{I} \\
& =\mathbf{A}\left(\begin{array}{cccc}
\sigma_{1}^{2} & & & \\
& \sigma_{2}^{2} & & \\
& & \ddots & \\
& & & \sigma_{D}^{2}
\end{array}\right) \mathbf{A}^{H}+\sigma_{n}^{2} \mathbf{I}
\end{aligned}
$$

Now, following [5], we vectorize $\mathbf{R}_{\mathbf{x x}}$ to get the following 
vector

$$
\begin{aligned}
\mathbf{z} & =\operatorname{vec}\left(\mathbf{R}_{\mathbf{x} \mathbf{x}}\right) \\
& =\operatorname{vec}\left[\sum_{i=1}^{D} \sigma_{i}^{2}\left(\mathbf{a}\left(\theta_{i}\right) \mathbf{a}^{H}\left(\theta_{i}\right)\right)\right]+\sigma_{n}^{2} \tilde{\mathbf{1}}_{n} \\
& =\left(\mathbf{A}^{*} \odot \mathbf{A}\right) \mathbf{p}+\sigma_{n}^{2} \tilde{\mathbf{1}}_{n}
\end{aligned}
$$

where $\mathbf{p}=\left[\begin{array}{ll}\sigma_{1}^{2} & \sigma_{2}^{2} \cdots \sigma_{D}^{2}\end{array}\right]^{T}$ and $\tilde{\mathbf{1}}_{n}=\left[\begin{array}{ll}\mathbf{e}_{\mathbf{1}}^{T} & \mathbf{e}_{\mathbf{2}}{ }^{T} \cdots \mathbf{e}_{\mathbf{N}}^{T}\end{array}\right]^{T}$ with $\mathbf{e}_{i}$ being a vector of all zeros except a 1 at the $i$ th position. Comparing it with (1), we can say that $\mathbf{z}$ in (3) behaves like the received signal at an array whose manifold is given by $\mathbf{A}^{*} \odot \mathbf{A}$. The equivalent source signal vector is represented by $\mathbf{p}$ and the noise becomes a deterministic vector given by $\sigma_{n}^{2} \tilde{\mathbf{1}}_{n}$. The distinct rows of $\mathbf{A}^{*} \odot \mathbf{A}$ behave like the manifold of a (longer) array whose sensor locations are given by the distinct values in the set $\left\{\overrightarrow{\mathbf{x}_{i}}-\overrightarrow{\mathbf{x}_{j}}, 1 \leq i, j \leq N\right\}$ where $\overrightarrow{\mathbf{x}}_{i}$ denotes the position vector of the $i$ th sensor of the original array. This array is precisely the difference co-array of the original array [10], [3]. So we can regard (3) as the new signal model and perform beamforming with respect to the signal powers instead of the signal amplitudes and this enables us to exploit fully the degrees of freedom of the difference co-array. To see this, consider taking the inner product of the vector $\mathbf{z}$ in (3) with a weight vector $\mathbf{w}$ to get

$$
\begin{aligned}
y & =\mathbf{w}^{H} \mathbf{z} \\
& =\sum_{i=1}^{D} \mathbf{w}^{H}\left(\mathbf{a}^{*}\left(\theta_{i}\right) \otimes \mathbf{a}\left(\theta_{i}\right)\right) \sigma_{i}^{2}+\sigma_{n}^{2} \mathbf{w}^{H} \tilde{\mathbf{1}}_{n}
\end{aligned}
$$

Defining the new beampattern as

$$
B_{\text {power }}(\theta)=\mathbf{w}^{H}\left(\mathbf{a}^{*}(\theta) \otimes \mathbf{a}(\theta)\right),
$$

we can write (4) as

$$
y=\sum_{i=1}^{D} B_{\text {power }}\left(\theta_{i}\right) \sigma_{i}^{2}+\sigma_{n}^{2} \mathbf{w}^{H} \tilde{\mathbf{1}}_{n}
$$

Hence the power of the $i$ th source from direction $\theta_{i}$ gets spatially filtered by the amount $B_{\text {power }}\left(\theta_{i}\right)$ and thereby spatial filtering (or beamforming) is performed with respect to the power of the signal. Note that though the beampattern spatially filters the power of the signal, the value of $B_{\text {power }}(\theta)$ can be, in general, complex. However, for detection purpose, we shall compare the value of $|y|$ with some appropriate threshold after constraining $B_{\text {power }}\left(\theta_{0}\right)$ to be 1 and making the contribution of terms due to the jammers and noise arbitrarily small. Therefore, the complex value of $B_{\text {power }}(\theta)$ will not affect the detection. As is evident, this kind of beamforming is based on a non linear preprocessing (taking the autocorrelation of the received signal vector) and hence it is essentially a non linear beamformer. It also requires uncorrelated sources and enough snapshots to realize the autocorrelation matrix.

In [7], we discuss the co-array perspective in greater detail and point out that the maximum degrees of freedom obtainable from the co-array of an $N$ element array possessing any geometry, is

$$
\mathrm{DOF}_{\text {max }}=N(N-1)+1 \text {. }
$$

However, in general, construction of arrays whose difference co-array can provide dramatic increase in degrees of freedom, is difficult and they are mostly found through computer search [11], [9]. To address this problem, we propose a novel class of arrays called the "nested arrays", which, as we shall demonstrate, can provide $O\left(N^{2}\right)$ degrees of freedom using only $O(N)$ physical sensors. The "two level" nested array as we shall define below, coincides with the array structure originally proposed by Bracewell (see [8]). However, we shall generalize the structure to beyond 2 levels to systematically increase the degrees of freedom.

\section{NESTED ARRAYS}

\section{A. 2 levels of nesting}

A two-level nested array is a concatenation of two ULAs: inner and outer where the inner ULA has $N_{1}$ elements with spacing $d_{1}$ and the outer ULA has $N_{2}$ elements with spacing $d_{2}$ such that $d_{2}=\left(N_{1}+1\right) d_{1}$. Fig. 1(a) shows an example. The following Lemma points out an important fact about its difference co-array.

Lemma 1: Let us consider a 2 level nested array with $N_{1}$ and $N_{2}$ elements in the two levels respectively. Then,

- Its difference co-array is a filled ULA.

- The difference co-array has $2 N_{2}\left(N_{1}+1\right)-1$ degrees of freedom with co-array elements located at

$$
\left\{n d_{1}, n=-M, \cdots, 0, \cdots, M\right\}
$$

where $M=N_{2}\left(N_{1}+1\right)-1$.

Fig.1(b) demonstrates the difference co-array of the two level nested array. We can further optimize the distribution of

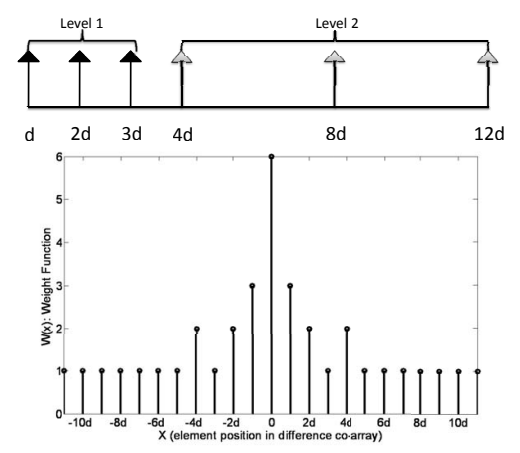

Fig. 1. A 2 level nested array with 3 sensors per level, and its difference co-array.

sensors in the two levels by finding $N_{1}, N_{2}$ that maximize the total degrees of freedom, $2 N_{2}\left(N_{1}+1\right)-1$, under the constraint of fixed total number of sensors, i.e., $N_{1}+N_{2}=N$. The solution (using AM-GM inequalities) can be verified as:

\begin{tabular}{|c|c|c|}
\hline $\mathrm{N}$ & optimal $N_{1}, N_{2}$ & DOF \\
\hline even & $N_{1}=N_{2}=\frac{1}{2} N$ & $\frac{N^{2}-2}{2}+N$ \\
\hline odd & $N_{1}=\frac{N-1}{2}, \quad N_{2}=\frac{N+1}{2}$ & $\frac{N^{2}-1}{2}+N$ \\
\hline
\end{tabular}

Hence, using 2 level of nesting, we can obtain approximately $N^{2} / 2$ degrees of freedom from $N$ elements. However this is approximately half of the maximum limit in (7). So now we examine how far we can increase the degrees of freedom of the co-array by extending the nesting strategy to more than two levels.

\section{B. K levels of Nesting}

A ' $K$-level' nested linear array, with $N_{i}$ sensors in the $i$ th level of nesting, is defined as one where the sensor positions 
are given by the set $\mathrm{S}_{(K \text {-level })}=\bigcup_{i=1}^{K} \mathrm{~S}_{i}$ where

$$
\begin{gathered}
\mathrm{S}_{i}=\left\{n d \prod_{j=1}^{i-1}\left(N_{j}+1\right), \quad n=1,2, \cdots, N_{i}\right\}, \quad i=2, \cdots, K \\
\mathrm{~S}_{1}=\left\{n d, \quad n=1, \cdots, N_{1}\right\}
\end{gathered}
$$

The following theorem which is proved in [7], gives the structure of the optimally nested array (i.e., the $K$-level nested array with maximum degrees of freedom) and its degrees of freedom:

Theorem 1: Given a number $N$ of sensors, the optimal number of nesting levels $K$ and the number of sensors per nesting level are given by

$$
\begin{gathered}
K=N-2 \\
N_{i}= \begin{cases}1, & i=1,2,3, \cdots, K-1 \\
2, & i=K\end{cases}
\end{gathered}
$$

and

The corresponding difference array is a non uniform linear array with degrees of freedom given by $\mathrm{DOF}_{\mathrm{opt}}=N(N-$ 1) +1 which is same as the upper bound in (7).

The nested arrays therefore provide an easy way to construct arrays whose difference co-arrays provide dramatic increase in degrees of freedom (close to the upper limit for large $N$ ). The sensor positions and expressions for degrees of freedom are exactly computable as a function of $N$ which is not possible for the existing classes of Minimum Redundancy arrays [9], [11].

We now exhibit some examples of different approaches to beamforming that can be performed with this array. Since the two-level nested array has a uniform difference co-array, we would concentrate on beamforming with 2 level nesting since all the conventional beamforming approaches are based on ULAs. However, the beamforming techniques might be extended to the optimally nested array as well.

\section{Beamforming with Nested Arrays}

\section{A. Deterministic Beamforming}

Let us consider a 2 level nested array with $N / 2$ sensors in each level. The difference co-array is an $U L A$ with $\left(N^{2}-\right.$ $2) / 2+N$ elements. Let $\mathbf{b}(\theta)$ denote the $\left(\left(N^{2}-2\right) / 2+N\right) \times 1$ steering vector of the ULA corresponding to the co-array. Say we want to realize a beam $\mathrm{B}_{\text {des }}(\theta)$ with this ULA and let $\mathbf{w}_{\text {des }}$ denote the deterministic weights such that

$$
\mathbf{w}_{\text {des }}^{H} \mathbf{b}(\theta)=\mathrm{B}_{\text {des }}(\theta) \text {. }
$$

Now to realize this beampattern with the two level nested array, let $\mathbf{w}$ denote the weight vector we would like to apply to the vector $\mathbf{z}$ in (3). Using (8), this means

$$
\mathbf{w}^{H}\left(\mathbf{a}^{*}(\theta) \otimes \mathbf{a}(\theta)\right)=\mathbf{w}_{\text {des }}^{H} \mathbf{b}(\theta), \quad \forall \theta .
$$

From the discussion of the co-array of the 2 level nested array in Section III, it can be easily seen that the $N^{2} \times 1$ vector $\mathbf{a}^{*}(\theta) \otimes \mathbf{a}(\theta)$ consists of the same rows as $\mathbf{b}(\theta)$, except the fact that some of them occur more than once. Let $n_{i}$ denote the number of times the $i$ th row of $\mathbf{b}(\theta)$ occurs in $\mathbf{a}^{*}(\theta) \otimes \mathbf{a}(\theta)$ and let $i_{1}, i_{2}, \cdots, i_{n_{i}}$ denote the row numbers where it occurs in $\mathbf{a}^{*}(\theta) \otimes \mathbf{a}(\theta)$. Then the weight vector $\mathbf{w}$ is given by

$$
\begin{aligned}
{[\mathbf{w}]_{i_{j}}=\frac{1}{n_{i}}\left[\mathbf{w}_{\text {des }}\right]_{i}, } & j=1,2, \cdots n_{i} \\
i & =1,2, \cdots,\left(N^{2}-2\right) / 2+N
\end{aligned}
$$

\section{B. Nulling of Jammers and Noise}

Consider $J$ jammers incident on the 2 level nested array from directions $\left\{\theta_{i}, i=1,2, \cdots, J\right\}$ respectively. Also assume that we are interested in looking into the direction $\theta_{0}$. Using the proposed beamformer in (8), this means we wish to determine the weight $\mathrm{w}$ to spatially null the jammers and maintain unity response in the look direction. This means

$$
B\left(\theta_{0}\right)=1, \quad B\left(\theta_{i}\right)=0, \quad i=1,2, \cdots, J .
$$

However, it can be noticed from (6) that the random noise now assumes the form of a deterministic vector $\tilde{\mathbf{1}}_{\mathbf{n}}$, which can also be nulled by the weight $\mathbf{w}$. Hence, using this beamforming, we can effectively null the noise term as well. This is something which really cannot be achieved by the conventional beamforming. Thus to null both jammers and noise, $\mathbf{w}$ should be selected as the solution to

$$
\left(\begin{array}{c}
\left(\mathbf{a}^{*}\left(\theta_{0}\right) \otimes \mathbf{a}\left(\theta_{0}\right)\right)^{H} \\
\left(\mathbf{a}^{*}\left(\theta_{1}\right) \otimes \mathbf{a}\left(\theta_{1}\right)\right)^{H} \\
\left(\mathbf{a}^{*}\left(\theta_{2}\right) \otimes \mathbf{a}\left(\theta_{2}\right)\right)^{H} \\
\vdots \\
\left(\mathbf{a}^{*}\left(\theta_{J}\right) \otimes \mathbf{a}\left(\theta_{J}\right)\right)^{H} \\
\left(\tilde{\mathbf{1}}_{\mathbf{n}}\right)^{T}
\end{array}\right) \mathbf{w}=\left(\begin{array}{c}
1 \\
0 \\
0 \\
\vdots \\
0 \\
0
\end{array}\right)
$$

With this beamforming, we can null noise, and upto $\left(N^{2}-\right.$ $2) / 2+N-1$ jammers, since we have in all $\left(N^{2}-2\right) / 2+N$ degrees of freedom in the co-array of the 2-level nested array. This technique for nulling jammers can also be extended to the case of the optimally nested array by simply replacing $\mathbf{a}(\theta)$ of the 2 level nested array with that of the optimally nested one in (10).

It is to be noted that in practice, since the signal covariance matrix is estimated from a finite number of snapshots, the kronecker product in (10) is only an approximation and hence (10) is satisfied only approximately. However, as we shall show in the numerical examples, the performance is quite satisfactory for moderate number of snapshots.

\section{Numerical EXAMPLES}

In this section we demonstrate the capability to perform beamforming with increased degrees of freedom offered by the 2 level nested array. We can envision two plots representing the beampattern. One way is to plot the pattern $B(\theta)$ as defined in (5) directly after computing the necessary weights. This however is not practical because the kronecker product in (5) is realized only approximately due to finite snapshots. Hence, for practical reason, to plot a more realistic beampattern, we consider a single source at angle $\theta$ and plot the amplitude response of our non linear beamformer to it by varying $\theta$ for finite number of snapshots. This automatically takes into consideration the finite sample effect. We call the former beam as ideal beampattern and the latter as practical beampattern.

\section{A. Realizing a Given Beampattern}

Let us consider a simple sinc beampattern obtained by applying uniform weighting to a 23 element uniform linear array.

Since a 6 element array $(N=6)$ with 2 level of nesting gives $\left(N^{2}-2\right) / 2+N=23$ degrees of freedom, we consider a 6 element array and plot both the ideal and practical beampatterns in Fig 2. The practical beampattern is generated using $T=100$ snapshots and it approximates the ideal beampattern quite closely. 


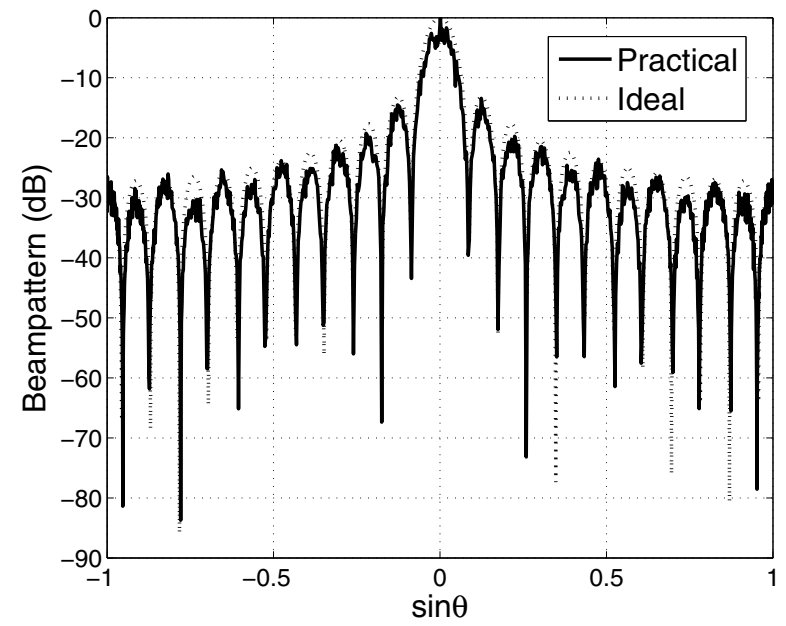

Fig. 2. Practical deterministic sinc beampattern obtained by non linear beamforming from the 2 level nested array, Number of sensors $N=6$, $T=100$.

\section{B. Beampattern for given jammer directions}

Consider a 4 element array with 2 levels of nesting. The look direction is $0^{\circ}$ and we consider 6 jammers from directions $\left\{-60^{\circ},-45^{\circ},-30^{\circ}, 15^{\circ}, 30^{\circ}, 45^{\circ}\right\}$ with jammer to signal power ratio $20 \mathrm{~dB}$ and signal to noise ratio $0 \mathrm{~dB}$. The ideal and practical beampatterns are generated using the weight computed from (10) and plotted in Fig 3. Note the introduction of nulls along the jammer positions. Also, the sidelobes are seen to be high which could pose problems for noise in the conventional beamforming. However, unlike conventional beamforming, in our case the noise has been reduced to a deterministic vector which can also be efficiently nulled. So the presence of noise at all spatial angles (which is assumed in conventional beamforming) can be ruled out and hence the high sidelobes do not pose a serious threat as far as noise is concerned. Also, we see that though this jammer and noise nulling theoretically requires perfect knowledge of signal covariance matrix, the practical beampattern shows that the approximation is reasonably well at moderate number of snapshots $(T=100)$.

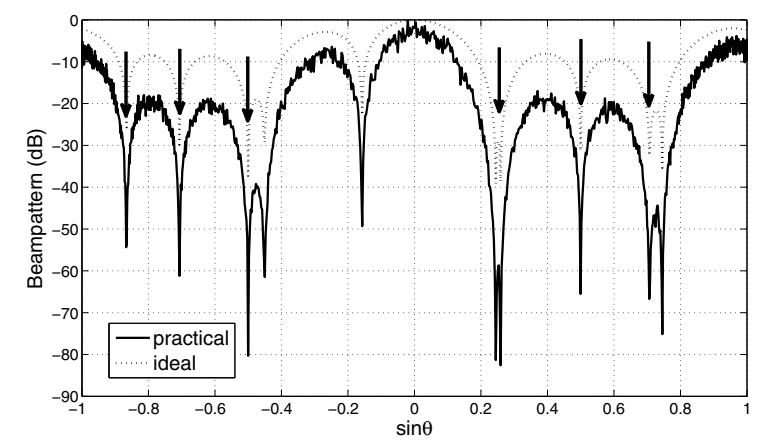

Fig. 3. Practical Beampattern obtained from the 2 level nested array after nulling 6 jammers and noise, Number of sensors $N=4, T=100$.

\section{CONCLUSION}

In this paper, we proposed a new approach to beamforming by suggesting a new nested array structure which provides an enormous increase in degrees of freedom. Instead of the signal amplitude, this beamforming spatially filters the powers of the impinging signals and hence it is essentially non linear in nature. It also has the capability of eliminating the noise provided enough snapshots are available. The performance of this beamformer is completely dependent on the model of the covariance matrix of the received signal and hence, in practical scenarios, the beamformer performance will keep improving with increasing number of snapshots. One immediate extension of this idea could be to demonstrate fully adaptive beamforming where the covariance matrix is updated with each incoming snapshot.

\section{REFERENCES}

[1] D. W. Bliss, and K.W. Forsythe, "Multiple-input multiple-output (MIMO) radar and imaging: Degrees of freedom and resolution", Proc. 37th IEEE Asilomar Conf. Signlas, Systems, Computers, vol. 1, pp. 54-59, Nov. 2003.

[2] Homer P. Bucker, "Cross-sensor beam forming with a sparse line array," J. Acoust. Soc. Am., Vol. 61, Issue 2, pp. 494-498, Feb. 1977.

[3] P. Chevalier, L. Albera, A. Ferreol, P. Comon, "On the virtual array concept for higher order array processing," IEEE Transactions on Signal Processing, vol. 53, no. 4, pp. 1254-1271, April 2005.

[4] Don H. Johnson and Dan E. Dudgeon, Array Signal Processing - Concepts and Techniques, Prentice-Hall, Englewood Cliffs, NJ, 1993.

[5] W. -K. Ma, T. -H. Hsieh, and C. -Y. Chi, "DOA estimation of quasi-stationary signals via Khatri-Rao subspace,", Proc. International Conference on Acoustics, Speech and Signal Processing (ICASSP), 2009, pp. 2165-2168, April 2009.

[6] Piya Pal, and P. P. Vaidyanathan, "A Novel Array Structure for Directions-of-Arrival Estimation with Increased Degrees of Freedom," International Conference on Acoustics, Speech and Signal Processing (ICASSP) 2010, Dallas, March 2010.

[7] Piya Pal, and P. P. Vaidyanathan, "Nested Arrays: A Novel approach to Array Processing with enhanced degrees of freedom," submitted to IEEE Transactions on Signal Processing.

[8] Hugh C. Pumphrey, "Design of Sparse Arrays in one, two and three dimensions," J. Acoust. Soc. Amer., vol. 93, Issue 3, pp. 1620-1628, March 1993

[9] H. L. Van Trees, Optimum Array Processing: Part IV of Detection, Estimation and Modulation Theory. New York: Wiley Interscience, 2002.

[10] R.T. Hoctor, S. A. Kassam, "The unifying role of the coarray in aperture synthesis for coherent and incoherent imaging," Proceedings of the IEEE, vol. 78, no. 4, pp. 735-752, April 1990.

[11] C. S., Ruf, "Numerical annealing of low-redundancy linear arrays," IEEE Transactions on Antennas and Propagation, vol. 41, no. 1, pp. 85-90, Jan. 1993. 